\title{
A database for medicinal and aromatic plants of JK (Jammu and Kashmir) in India
}

\author{
Akbar Masood* and Mujtaba Shafi \\ Bioinformatics Centre, The University of Kashmir, Hazratbal, Srinagar, Jammu and Kashmir, India-190006; \\ Akbar Masood * - Email: akbar@bioinfoku.org; * Corresponding author
}

received September 26, 2005; revised October 6, 2005; accepted October 6, 2005; published online October 7, 2005

\begin{abstract}
:
High throughput screening of small molecules for a given drug target is achieved using plant materials of medicinal value. Therefore, it is important to document the availability and location of such medicinal plants in the form of a database. Here, we describe a web database containing information (botanical name, common name, local name, botany, chemistry, folklore medicinal use and medicinal uses) about the medicinal and aromatic plants available in JK (Jammu and Kashmir). The database is available for free in public domain.
\end{abstract}

Availability: http://www.bioinfoku.org/db/medsearch.php

Keywords: medicinal; aromatic; plants; Jammu and Kashmir; India; botanical name; common name; folklore

\section{Background:}

Medicinal plants documented in the literature from thick vegetations of the world are routinely used for high throughput screening in small molecular drug discovery. A large number of such plants are known to be recognized in a discrete manner. Therefore, it is important to store information related to medicinal and aromatic plants of JK in a database. This will help in the use and exploitation of the plant materials for drug discovery. Some of the plants available in this region are also known to have aromatic value. Here, we describe the development and use of a database containing information on medicinal and aromatic plants from JK.

\section{Methodology:}

Dataset:

Folklore medicinal usage and other associated data were manually collected from the inhabitants (tribal/gujjars/local clinicians) of JK through direct person to person survey for each plant species. The current dataset contains information for about 133 plant species.
Database Interface:

The database interface is designed for searching the dataset using a PHP (a programming language that allows web developers to create dynamic content that interacts with databases) server enabled script for keywords such as botanical name, local name, folklore use and medicinal use. The database is accessible for free through the internet.

\section{Database Design:}

The database is developed in MySQL on a Linux Platform and updated regularly.

\section{Search result:}

The search output for a given query (for example, diabetes) is given in Figure 1.

Utility:

The database finds utility in the selection of medicinal plant species for high throughput screening in drug discovery.

\section{Acknowledgement:}

This project is funded by BTIS (Biotechnology information system), DBT (Department of Biotechnology), Ministry of Science and Technology, Government of India, India. 


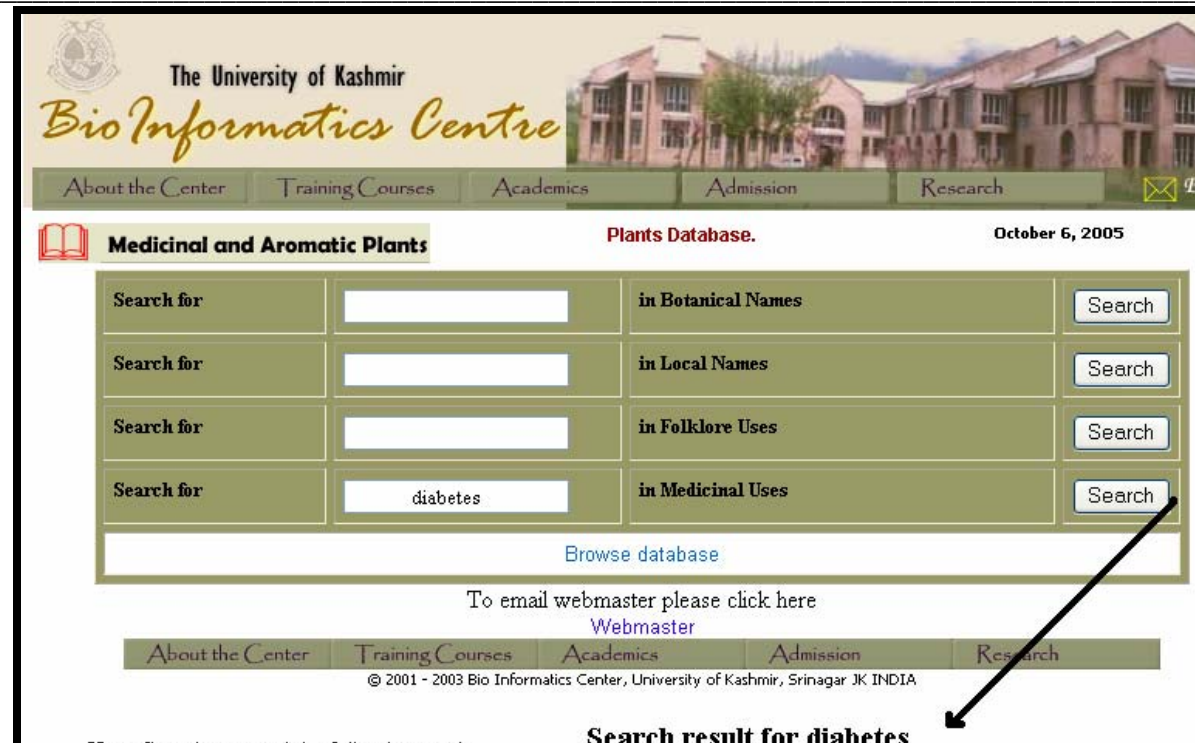

Your Search returned the following results.

Search result for diabetes

Click on the plant name to see more details.

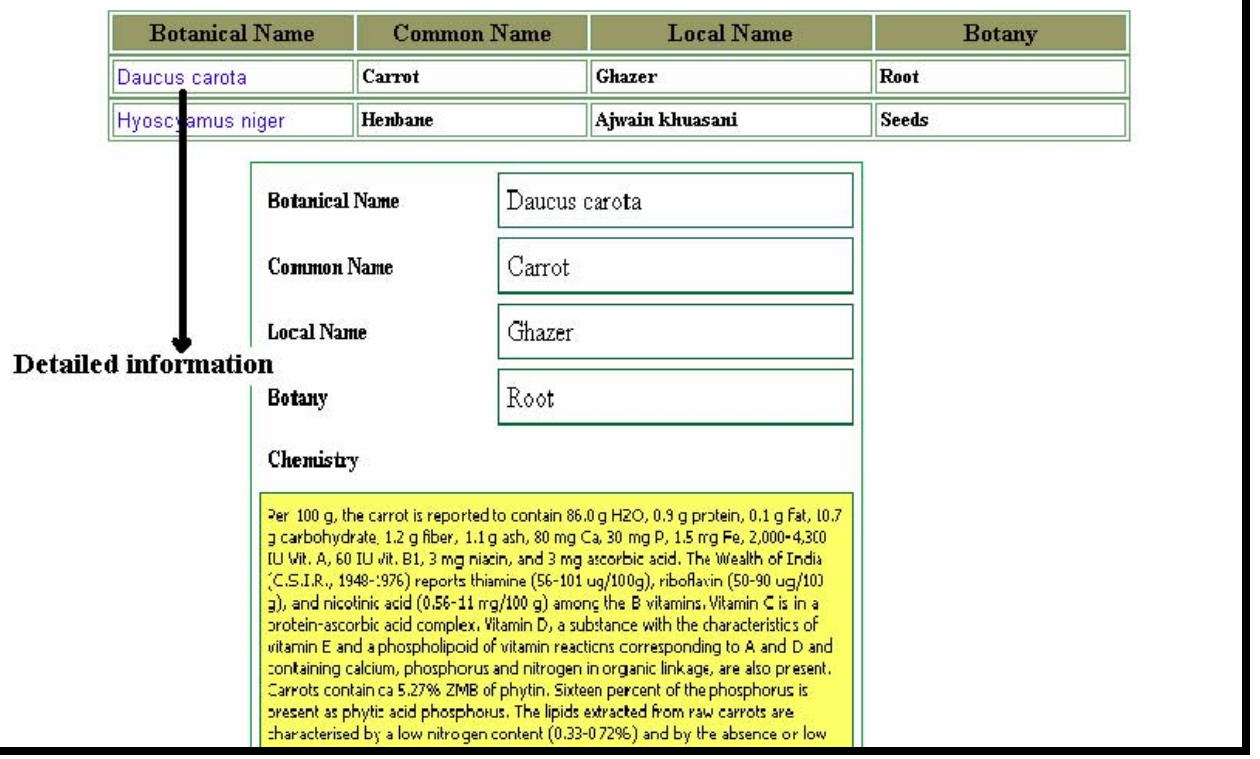

Figure 1: A database for medicinal and aromatic plants of JK (Jammu and Kashmir) in India. A sample output for 'diabetes' is shown

Edited by P. Kangueane

Citation: Masood \& Shafi, Bioinformation 1(2): 56-57 (2005)

License statement: This is an open-access article, which permits unrestricted use, distribution, and reproduction in any medium, for non-commercial purposes, provided the original author and source are credited. 